PROCEEDINGS OF THE

AMERICAN MATHEMATICAL SOCIETY

Volume 130, Number 12, Pages 3701-3711

S 0002-9939(02)06465-1

Article electronically published on April 22, 2002

\title{
PERFECTLY MEAGER SETS AND UNIVERSALLY NULL SETS
}

\author{
TOMEK BARTOSZYNSKI AND SAHARON SHELAH
}

(Communicated by Alan Dow)

\begin{abstract}
We will show that there is no ZFC example of a set distinguishing between universally null and perfectly meager sets.
\end{abstract}

\section{INTRODUCTION}

Consider the following three families of sets of reals:

Definition 1. Let $X \subseteq \mathbb{R}$. Then:

(1) $X$ is perfectly meager if for every perfect set $P \subseteq \mathbb{R}, P \cap X$ is meager in $P$.

(2) $X$ is universally meager if every Borel isomorphic image of $X$ is meager.

(3) $X$ is universal null if every Borel isomorphic image of $X$ has Lebesgue measure zero.

Let $\mathbf{P M}, \mathbf{U M}$ and $\mathbf{U N}$ denote these families respectively.

The family UM was studied recently by Zakrzewski [13, and identified as an analog of UN.

One gets an equivalent definition of UN by replacing "Borel isomorphic" with "homeomorphic", but this is not the case with UM.

Let $\mathcal{M}$ and $\mathcal{N}$ denote the $\sigma$-ideals of meager and of measure zero subsets of the reals, respectively.

For a $\sigma$-ideal $\mathcal{J} \subseteq P(\mathbb{R})$ let

$$
\operatorname{non}(\mathcal{J})=\min \{|X|: X \subseteq \mathbb{R} \& X \notin \mathcal{J}\} .
$$

There are many ZFC examples of uncountable sets that are in $\mathbf{U M} \cap \mathbf{U N}$. These include $\omega_{1} \omega_{1}^{\star}$-gaps, a selector from the constituents of a non-Borel $\boldsymbol{\Pi}_{1}^{1}$ set, etc. (see 9]). All these sets have size $\aleph_{1}$, since Miller [8] showed that, consistently, no set of size $2^{\aleph_{0}}$ is in $\mathbf{U M} \cup \mathbf{U N}$.

Grzegorek found other constructions in ZFC that produce sets of (consistently) different sizes.

Theorem 2 (Grzegorek, [6]). (1) There exists a set $X \in \mathbf{U N}$ such that $|X|=$ $\operatorname{non}(\mathcal{N})$,

(2) There exists a set $X \in \mathbf{U M}$ such that $|X|=\operatorname{non}(\mathcal{M})$.

Received by the editors April 26, 2001 and, in revised form, July 16, 2001

2000 Mathematics Subject Classification. Primary 03E17.

Key words and phrases. Perfectly meager, universally null, consistency.

The first author was partially supported by NSF grant DMS 9971282 and the Alexander von Humboldt Foundation.

The second author was partially supported by the Israel Science Foundation. Publication 732 .

(C)2002 American Mathematical Society 
The problem of whether the equality $\mathbf{U M}=\mathbf{U N}$ is consistent is open. However, both inclusions are consistent with ZFC; UM $\subsetneq$ UN holds in a model obtained by adding $\boldsymbol{\aleph}_{2}$ Cohen reals, and $\mathbf{U N} \subsetneq \mathbf{U M}$ holds in a model obtained by adding $\boldsymbol{\aleph}_{2}$ random reals (side-by-side) (see [4], [9], [8]).

In this paper we investigate the connection between families $\mathbf{U N}$ and $\mathbf{P M}$, and show that both inclusions $\mathbf{P M} \subseteq \mathbf{U N}$ and $\mathbf{U N} \subseteq \mathbf{P M}$ are consistent with ZFC as well. Observe that trivially $\mathbf{U M} \subseteq \mathbf{P M}$, thus we only need to check that $\mathbf{P M} \subseteq \mathbf{U N}$ is consistent. Recall that $\mathbf{P M} \neq \mathbf{U M}$ is consistent ([12]) as well as $\mathbf{P M}=\mathbf{U M}([2])$. We will show that:

Theorem 3. It is consistent with ZFC that $\mathbf{P M} \subseteq[\mathbb{R}] \leq \boldsymbol{\aleph}_{1} \subseteq \mathbf{U N}$.

\section{Forcing}

Suppose that $X \subseteq 2^{\omega}$ is a perfectly meager set in $\mathbf{V}$. Let $\widetilde{P}$ be a fixed closed subset of $2^{\omega} \times 2^{\omega}$ which is universal for perfect sets in $2^{\omega}$. In other words, for every perfect set $P \subseteq 2^{\omega}$ there exists an $x$ such that $P=(\widetilde{P})_{x}=\{y:(x, y) \in \widetilde{P}\}$. Note that this property is absolute. Since $X$ is perfectly meager, we can find sets $\widetilde{Q^{n}} \subseteq 2^{\omega} \times 2^{\omega}$ such that for every $x \in 2^{\omega}$ and $n \in \omega$,

(1) $\left(\widetilde{Q^{n}}\right)_{x}$ is a closed nowhere dense subset of $(\widetilde{P})_{x}$,

(2) $X \cap(\widetilde{P})_{x} \subseteq\left(\bigcup_{n \in \omega} \widetilde{Q^{n}}\right)_{x}$.

Clearly, the set $\bigcup_{n \in \omega} \widetilde{Q^{n}}$ witnesses that $X \in \mathbf{P M}$ since

$$
X \subseteq 2^{\omega} \backslash \bigcup_{x \in 2^{\omega}}\left(\widetilde{P} \backslash \bigcup_{n \in \omega} \widetilde{Q^{n}}\right)_{x} .
$$

Note that the last inclusion makes sense even if $X$ is not a subset of $\mathbf{V}$. Suppose that $\mathbf{V}^{\prime} \subseteq \mathbf{V}$ and $X \subseteq \mathbf{V}$ is a set of reals. We will say $\mathbf{V}^{\prime} \models X \in \mathbf{P M}$ if there exists a family $\left\{\widetilde{Q^{n}}: n \in \omega\right\} \in \mathbf{V}^{\prime}$ such that $X \cap(\widetilde{P})_{x} \subseteq\left(\bigcup_{n \in \omega} \widetilde{Q^{n}}\right)_{x}$ for every real $x \in \mathbf{V}^{\prime}$.

The property of being perfectly meager is not absolute, so whether $X$ is perfectly meager in $\mathbf{V}^{\prime}$ has no bearing onto whether $X$ is perfectly meager in $\mathbf{V}$. For example, if $x \in \mathbf{V}$ is a Cohen real over $\mathbf{V}^{\prime}$, then the set $\{x\}$ is perfectly meager in $\mathbf{V}$ but not in $\mathbf{V}^{\prime}$.

Lemma 4. Let $\left\{\mathcal{P}_{\alpha}, \dot{\mathcal{Q}}_{\alpha}: \alpha<\omega_{2}\right\}$ be a countable support iteration of proper forcing notions over $\mathbf{V} \models \mathrm{CH}$. Suppose that $X \subseteq \mathbf{V}^{\mathcal{P}_{\omega_{2}}} \cap \mathbb{R}$ is a perfectly meager set. Then there exists an $\omega_{1}$-club $C \subseteq \omega_{2}$ such that for every $\alpha \in C$,

$$
\mathbf{V}^{\mathcal{P}_{\alpha}}=X \in \mathbf{P M}
$$

Proof. Let $\left\{\widetilde{Q^{n}}: n \in \omega\right\} \in \mathbf{V}^{\mathcal{P}_{\omega_{2}}}$ be a family witnessing that $X$ is perfectly meager. Let $C$ consist of those ordinals of cofinality $\omega_{1}$ such that $\widetilde{Q^{n}} \cap\left(\left(2^{\omega} \cap \mathbf{V}^{\mathcal{P}_{\alpha}}\right) \times 2^{\omega}\right) \in$ $\mathbf{V}^{\mathcal{P}_{\alpha}}$ for every $n$. The usual argument involving Skolem-Löwenheim theorem shows that $C$ has the required property.

Our objective is to find a set of general conditions on a forcing notion $\mathbb{P}$ such that the countable support iteration of $\mathbb{P}$ of length $\omega_{2}$ produces a model where $\mathbf{P M} \subseteq[\mathbb{R}]^{\leq \boldsymbol{\aleph}_{1}} \subseteq \mathbf{U N}$. These conditions are sufficient for the class of forcing notions defined using norms [10]. 
These conditions are the following:

(1) $\mathbf{V}^{\mathbb{P}}=\mathbf{V} \cap 2^{\omega} \in \mathcal{N}$

(2) $\mathbf{V}^{\mathbb{P}} \models \mathbf{V} \cap 2^{\omega} \notin \mathcal{M}$,

(3) $\mathbb{P}$ is $\omega^{\omega}$-bounding, that is, $\omega^{\omega} \cap \mathbf{V}$ is a dominating family in $\omega^{\omega} \cap \mathbf{V}^{\mathbb{P}}$,

(4) $\mathbb{P}$ adds a real $x_{\mathbb{P}} \in 2^{\omega}$ such that $\mathbf{V} \models\left\{x_{\mathbb{P}}\right\} \notin \mathbf{P M}$,

(5) $\mathbb{P}$ generic real is minimal, that is, if $g$ is $\mathbb{P}$-generic over $\mathbf{V}$ and $x \in \mathbf{V}[g] \cap 2^{\omega}$, then $x \in \mathbf{V}$ or $g \in \mathbf{V}[x]$.

Condition (1) is necessary to make all sets of size $\boldsymbol{\aleph}_{1}$ universally null, and condition (2) is necessary to avoid making all $\aleph_{1}$ sets perfectly meager. Recall that (2) and (3) together are essentially equivalent to $\mathbf{V}^{\mathbb{P}} \models \mathbf{V} \cap \mathcal{M}$ is cofinal in $\mathcal{M}$.

For the forcing notions $\mathbb{P}$ that we have in mind the following property holds: for every real $x \in \mathbf{V}^{\mathbb{P}}$ there exists a continuous function $f \in \mathbf{V}$ such that $x=f\left(x_{G}\right)$, where $x_{G}$ is a generic real.

Condition (5) guarantees that in the above context $f$ can be chosen to be a homeomorphism. In particular, if $X$ is a set of reals of size $\aleph_{2}$, then $X$ will contain a homeomorphic image of a sequence of generic reals.

The following forcing notion appeared in [5]; it is similar (but not identical) to the infinitely equal real forcing from 7 .

For a tree $p$ and $t \in p$, let $\operatorname{succ}_{p}(t)$ be the set of all immediate successors of $t$ in $p$, $p_{t}=\{v \in p: t \subseteq v$ or $v \subseteq t\}$ the subtree of $p$ determined by $t, p\lceil n$ the $n$-th level of $p$, and $[p]$ the set of branches of $p$. By identifying $s \in \omega^{<\omega}$ with the full-branching tree having root $s$, we can also denote $[s]=\left\{f \in \omega^{\omega}: s \subseteq f\right\}$.

Fix a strictly increasing function $f \in \omega^{\omega}$ and let $\mathbf{X}=\prod_{n \in \omega} f(n)$. Note that $\mathbf{X}$ is a Polish space homeomorphic to $2^{\omega}$. For technical reasons we require that $f(n)=2^{\tilde{f}(n)}$ for $n \in \omega$.

Let $\mathbb{E} \mathbb{E}$ be the following forcing notion: $p \in \mathbb{E} \mathbb{E}$ if

(1) $p$ is a nonempty subtree of $\omega^{<\omega}$,

(2) $s(n)<f(n)$ for all $s \in p$ and $n \in \operatorname{dom}(s)$,

(3) for all $s \in p$ there exists an extension $t$ of $s$ such that $t^{\complement} n \in p$ for all $n<f(|t|)$.

For $p, q \in \mathbb{E} \mathbb{E}, p \geq q$ if $p \subseteq q$. Without loss of generality we can assume that $\left|\operatorname{succ}_{p}(s)\right|=1$ or $\operatorname{succ}_{p}(s)=f(|p|)$ for all $p \in \mathbb{E} \mathbb{E}$ and $s \in p$. Conditions of this type form a dense subset of $\mathbb{E} \mathbb{E}$. Let

$$
\operatorname{split}(p)=\left\{s \in p:\left|\operatorname{succ}_{p}(s)\right|>1\right\}=\bigcup_{n \in \omega} \operatorname{split}_{n}(p),
$$

where $\operatorname{split}_{n}(p)=\{s \in \operatorname{split}(p):|\{t \subsetneq s: t \in \operatorname{split}(p)\}|=n\}$.

For $p, q \in \mathbb{E} \mathbb{E}, n \in \omega$, we let

$$
p \geq_{n} q \Longleftrightarrow p \geq q \& \operatorname{split}_{n}(q)=\operatorname{split}_{n}(p) \text {. }
$$

Lemma 5 ([5]). (1) $\mathbb{E} \mathbb{E}$ satisfies Axiom $A$, so it is proper,

(2) $\mathbf{V}^{\mathbb{E E}} \models \mathbf{V} \cap 2^{\omega} \in \mathcal{N}$,

(3) $\mathbf{V}^{\mathbb{E E}} \models \mathbf{V} \cap 2^{\omega} \notin \mathcal{M}$,

(4) for every maximal antichain $\mathcal{A} \subseteq \mathbb{E} \mathbb{E}, p \in \mathbb{E} \mathbb{E}$, and $n \in \omega$ there exists $q \geq_{n} p$ such that $\{r \in \mathcal{A}: r$ is compatible with $q\}$ is finite,

(5) for every family of maximal antichains $\left\{\mathcal{A}_{n}: n \in \omega\right\}$ and $p \in \mathbb{E} \mathbb{E}$ there exists $q \geq p$ such that for every $n,\left\{r \in \mathcal{A}_{n}: r\right.$ is compatible with $\left.q\right\}$ is finite,

(6) $\mathbb{E} \mathbb{E}$ is $\omega^{\omega}$ bounding,

(7) $\mathbf{V}^{\mathbb{E E}}=\mathbf{V} \cap \mathcal{M}$ is cofinal in $\mathcal{M}$. 
Note that for $p \in \mathbb{E} \mathbb{E}$ the set $[p]$ is a compact subset of $\mathbf{X}=\prod_{n} f(n)$. Moreover, there is a canonical isomorphism between $[p]$ and $2^{\omega}$ defined as follows:

For every $n$ let $\left\{s_{0}^{n}, \ldots, s_{f(n)}^{n}\right\}$ be a fixed enumeration of $0-1$ sequences of length $\tilde{f}(n)$ (recall that $\left.f(n)=2^{\tilde{f}(n)}\right)$. Define $F:[p] \longrightarrow 2^{\omega}$ as

$$
F(x)=s_{x\left(n_{0}+1\right)}^{n_{0}} \frown s_{x\left(n_{1}+1\right)}^{n_{1}} \frown \ldots,
$$

where $n_{0}, n_{1}, \ldots$ is the increasing enumeration of the set $\{n: x\lceil n \in \operatorname{split}(p)\}$.

Lemma 6. Let $p \in \mathbb{E} \mathbb{E}$ and suppose that $H \subseteq[p]$ is a meager set in $[p]$. For every $n \in \omega$ there exists $q \geq_{n} p$ such that $[q] \cap H=\emptyset$. In particular, $\Vdash_{\mathbb{E E}}$ "V $\models\{\dot{g}\} \notin$ PM".

Proof. Let $H \subseteq[p]$ be a meager set, and let $n \in \omega$. Fix a descending sequence of open sets $\left\langle U_{k}: k \in \omega\right\rangle$ such that each $U_{k}$ is dense in $[p]$ and $H \cap \bigcap_{k} U_{k}=\emptyset$. By induction build a sequence $\left\langle p_{k}: k \in \omega\right\rangle$ such that $p_{0}=p$, and for every $k$,

(1) $p_{k+1} \geq_{n+k+1} p_{k} \in \mathbb{E} \mathbb{E}$,

(2) $\left[p_{k+1}\right] \subseteq U_{k}$.

Suppose that $p_{k}$ is given. For every $v \in$ split $_{n+k+1}\left(p_{k}\right)$ find $q_{v} \geq\left(p_{k}\right)_{v}$ such that $\left[q_{v}\right] \subseteq U_{k}$. Let $p_{k+1}=\bigcup\left\{q_{v}: v \in \operatorname{split}_{n+k+1}\left(p_{k}\right)\right\}$. Condition $q=\lim _{k} p_{k}$ has the required property.

Suppose that $\left\{\widetilde{Q^{n}}: n \in \omega\right\} \in \mathbf{V}$ is a possible witness that $\{\dot{g}\}$ is perfectly meager, and let $p \in \mathbb{E} \mathbb{E}$. Find $x \in \mathbf{V}$ such that $[p]=(P)_{x}$ and let $q \geq p$ be such that $[q] \cap\left(\bigcup_{n} \widetilde{Q^{n}}\right)_{x}=\emptyset$. Clearly,

$$
q \Vdash_{\mathbb{E E}}\{\dot{g}\} \in \bigcup_{x \in \mathbf{V}}\left(P \backslash \bigcup_{n} \widetilde{Q^{n}}\right)_{x} .
$$

In particular, $q \Vdash_{\mathbb{E} \mathbb{E}}$ "V $\models\{\dot{g}\} \notin \mathbf{P M}$ ".

Lemma 7. Suppose that $p \in \mathbb{E} \mathbb{E}$ and $p \Vdash_{\mathbb{E E}} \dot{x} \in 2^{\omega}$. For every $n \in \omega$ there exist $q \geq_{n} p$ and a continuous function $F:[q] \longrightarrow 2^{\omega}$ such that $q \Vdash_{\mathbb{E E}} \dot{x}=F(\dot{g})$, where $\dot{g}$ is the canonical name for the generic real.

Moreover, we can require that for every $v \in \operatorname{split}_{n}(q)$ and any $x_{1}, x_{2} \in\left[q_{v}\right]$, $F\left(x_{1}\right)\left\lceil n=F\left(x_{2}\right)\lceil n\right.$.

Proof. The first part is a special case of a more general fact. For $n \in \omega$ let $\mathcal{A}_{n} \subseteq \mathbb{E} \mathbb{E}$ be a maximal antichain below $p$ such that $\forall r \in \mathcal{A}_{n} \exists s \in 2^{n} r \Vdash_{\mathbb{E E}}$ $\dot{x}\left\lceil n=s\right.$. Use Lemma 5 (5) to find $q \geq p$ such that for every $n \in \omega,\left\{r \in \mathcal{A}_{n}\right.$ : $r$ is compatible with $q\}$ is finite. Let $\mathcal{A}_{n}^{\prime}=\left\{r \in \mathcal{A}_{n}: r\right.$ is compatible with $\left.q\right\}$. Without loss of generality we can assume that $[q] \subseteq \bigcup_{r \in \mathcal{A}_{n}^{\prime}}[r]$. It follows that $[r] \cap[q]$ is clopen in $[q]$ for every $r \in \mathcal{A}_{n}^{\prime}$. Define $F:[q] \longrightarrow 2^{\omega}$ as $F(x)=y$ if for every $n \in \omega$ there exists $r \in \mathcal{A}_{n}^{\prime}$ such that $x \in[r]$ and $r \Vdash_{\mathbb{E E}} \dot{x}\lceil n=y\lceil n$. It is easy to see that $F$ is a continuous function that has the required properties.

To show the second part we need to build $q$ in such a way that for every $v \in$ $\operatorname{split}_{n}(q)$, there is $r \in \mathcal{A}_{n}^{\prime}$ such that $q_{v} \geq r$.

Lemma 8. Suppose that $p \in \mathbb{E} \mathbb{E}, n \in \omega$ and $p \Vdash_{\mathbb{E E}} \dot{x} \in 2^{\omega}$. Let $F:[q] \longrightarrow 2^{\omega}$ be a continuous function such that $p \Vdash_{\mathbb{E E}} \dot{x}=F(\dot{g})$.

There exists $q \geq p$ such that $F\left\lceil[q]\right.$ is constant, or there exists $q \geq_{n} p$ such that $F \uparrow[q]$ is one-to-one. In particular, the generic real is minimal. 
Proof. Consider the following two cases:

CASE 1. $p \|_{\mathbb{E E}} \dot{x} \notin \mathbf{V}$. Let $x \in \mathbf{V}$ and $q \geq p$ be such that $q \Vdash_{\mathbb{E E}} \dot{x}=x$. Clearly $F\lceil[q]$ is constant with value $x$.

CASE 2. $p \Vdash_{\mathbb{E E}} \dot{x} \notin \mathbf{V}$.

Build by induction a sequence of conditions $\left\langle p_{k}: k \in \omega\right\rangle$ such that $p_{0}=p$ and for every $k$,

(1) $p_{k+1} \geq_{n+k+1} p_{k}$,

(2) sets $\left\{F^{\prime \prime}\left(\left[\left(p_{k+1}\right)_{s}\right]\right): s \in\right.$ split $\left._{n+k+1}\left(p_{k+1}\right)\right\}$ are pairwise disjoint and have diameter $<2^{-k}$.

Suppose that $p_{k}$ is given. Note that $F^{\prime \prime}\left(\left[\left(p_{k}\right)_{s}\right]\right)$ is uncountable for every $s \in p_{k}$. For $v \in \operatorname{split}_{n+k+1}\left(p_{k}\right)$ choose pairwise different reals $x_{v} \in F^{\prime \prime}\left(\left[\left(p_{k}\right)_{v}\right]\right)$. It is not important now but will be relevant in the sequel, that we can choose these reals "effectively" from a fixed countable subset of $\left[p_{k}\right]$. Let $\ell>k$ be such that sequences $x_{v}\left\lceil\ell\right.$ are also pairwise different. For every $v \in \operatorname{split}_{n+k+1}\left(p_{k}\right)$ let $s_{v} \in \operatorname{split}\left(p_{k}\right)$ be such that for every $z \in\left[\left(p_{k}\right)_{s_{v}}\right], F(z)\left\lceil\ell=x_{v}\lceil\ell\right.$. If $F$ is as in the second part of Lemma 7, then we can find $s_{v}$ in $\operatorname{split}_{\ell}\left(p_{k}\right)$. Define $p_{k+1}=\bigcup\left\{\left(p_{k}\right)_{s_{v}}: v \in\right.$ split $\left._{n+k+1}\left(p_{k}\right)\right\}$. Observe that $q=\lim _{k} p_{k}$ has the required property.

Note that the above lemma shows that the reals added by $\mathbb{E} \mathbb{E}$ are minimal. Infinitely equal forcing from [7] or [4] does not have this property.

\section{ItERATION OF $\mathbb{E} \mathbb{E}$}

Let $\alpha \leq \omega_{2}$ be an ordinal and suppose that $\mathbb{E}_{\alpha}$ is a countable support iteration of $\mathbb{E} \mathbb{E}$ of length $\alpha$. In other words, $p \in \mathbb{E}_{\alpha}$ is

(1) $p$ is a function and $\operatorname{dom}(p)=\alpha$,

(2) $\operatorname{supp}(p)=\{\beta: p(\beta) \neq \emptyset\}$ is countable,

(3) $\forall \beta<\alpha p\left\lceil\beta \Vdash_{\mathbb{E E}_{\beta}} p(\beta) \in \mathbb{E} \mathbb{E}\right.$.

For $F \in[\alpha]^{<\omega}, n \in \omega$, and $p, q \in \mathbb{E E}_{\alpha}$ define

$$
q \geq_{F, n} p \Longleftrightarrow q \geq p \& \forall \beta \in F q \uparrow \beta \Vdash_{\mathbb{E E}_{\beta}} q(\beta) \geq_{n} p(\beta) .
$$

The following fact is well-known.

Theorem 9 ([5], 7], [3]). Suppose that $p \in \mathbb{E}_{\alpha}, F \in[\alpha]^{<\omega}$, and $n \in \omega$. Then:

(1) for every maximal antichain $\mathcal{A} \subseteq \mathbb{E}_{\alpha}$, there exists $q \geq_{F, n} p$ such that $\{r \in \mathcal{A}: r$ is compatible with $q\}$ is finite,

(2) for every family of maximal antichains $\left\{\mathcal{A}_{n}: n \in \omega\right\}$ there exists $q \geq p$ such that for every $n,\left\{r \in \mathcal{A}_{n}: r\right.$ is compatible with $\left.q\right\}$ is finite,

(3) $\mathbf{V}^{\mathbb{E E}_{\omega_{2}}}=[\mathbb{R}]^{<2^{\aleph_{0}}} \subseteq \mathcal{N}$,

(4) $\mathbf{V}^{\mathbb{E} \mathbb{E}_{\omega_{2}}} \models \mathcal{M} \cap \mathbf{V}$ is cofinal in $\mathcal{M}$.

For $p \in \mathbb{E E}_{\alpha}$ let $\operatorname{cl}(p)$ be the smallest set $w \subseteq \alpha$ such that $p$ can be evaluated using generic reals $\left\langle\dot{g}_{\beta}: \beta \in w\right\rangle$. In other words, $\mathrm{cl}(p)$ consists of those $\beta<\alpha$ such that the transitive closure of $p$ contains an $\mathbb{E E}_{\beta}$-name for an element of $\mathbb{E} \mathbb{E}$. It is well-known [11] that $\left\{p \in \mathbb{E E}_{\alpha}: \mathrm{cl}(p) \in[\alpha]^{\leq \omega}\right\}$ is dense in $\mathbb{E}_{\alpha}$. 
Suppose that $p \in \mathbb{E E}_{\alpha}, w=\operatorname{cl}(p)$ is countable and $\alpha_{p}=\operatorname{ot}(\operatorname{cl}(p))$. Let $\mathbb{E} \mathbb{E}_{w}$ be the countable support iteration of $\mathbb{E} \mathbb{E}$ with the domain $w$. In other words, consider the countable support iteration $\left\langle\mathcal{P}_{\beta}, \dot{\mathcal{Q}}_{\beta}: \beta<\sup (w)\right\rangle$ such that

$$
\forall \beta<\sup (w) \Vdash_{\mathcal{P}_{\beta}} \dot{\mathcal{Q}}_{\beta} \simeq \begin{cases}\mathbb{E} \mathbb{E} & \text { if } \beta \in w, \\ \emptyset & \text { if } \beta \notin w .\end{cases}
$$

It is clear that $\mathbb{E} \mathbb{E}_{w} \simeq \mathbb{E}_{\alpha_{p}}$. Moreover, we can view condition $p$ as a member of $\mathbb{E E}_{w}$.

For the rest of the section we will consider only the iteration of $\mathbb{E} \mathbb{E}$ of countable length $\alpha$ and show that $\mathbb{E} \mathbb{E}_{\alpha}$ has the same properties as $\mathbb{E} \mathbb{E}$.

Let $\alpha$ be a countable ordinal and $p \in \mathbb{E E}_{\alpha}$. Define $\bar{p} \subseteq \mathbf{X}^{\alpha}$ as follows:

$\left\langle x_{\beta}: \beta<\alpha\right\rangle \in \bar{p}$ if for every $\beta<\alpha$,

$$
x_{\beta} \in\left[p(\beta)\left[\left\langle x_{\gamma}: \gamma<\beta\right\rangle\right]\right] .
$$

Note that $p(\beta)\left[\left\langle x_{\gamma}: \gamma<\beta\right\rangle\right]$ is the interpretation of $p(\beta)$ using reals $\left\langle x_{\gamma}: \gamma<\beta\right\rangle$ so it may be undefined if these reals are not sufficiently generic.

For a set $G \subseteq \mathbf{X}^{\alpha}, u \subseteq \alpha$, and $x \in \mathbf{X}^{u}$ let

$$
(G)_{x}=\left\{y \in \mathbf{X}^{\alpha \backslash u}: \exists z \in G z\lceil u=x \& z\lceil(\alpha \backslash u)=y\},\right.
$$

and for $\beta \in \alpha$ let $(G)_{\beta}=\{x(\beta): x \in G\}$.

We say that $p \in \mathbb{E E}_{\alpha}$ is good if

(1) $\bar{p}$ is compact,

(2) for every $\beta<\alpha$ and $x \in \overline{p\lceil\beta}, \overline{p[x]}=(p)_{x}$ and $\overline{p(\beta)[x]}=\left((p)_{x}\right)_{\beta}$,

(3) $\bar{p}$ is homeomorphic to $\mathbf{X}^{\alpha}$ via a homeomorphism $h$ such that for every $\beta<\alpha$ and $x \in \overline{p\lceil\beta}, h \uparrow\left((p)_{x}\right)_{\beta}$ is a homeomorphism between $\left((p)_{x}\right)_{\beta}$ and $\mathbf{X}$.

Lemma 10. $\left\{p \in \mathbb{E}_{\alpha}: \bar{p}\right.$ is good $\}$ is dense in $\mathbb{E}_{\alpha}$.

Proof. CASE 1. $\alpha=\beta+1$.

Fix $p \in \mathbb{E}_{\alpha}$ and for $n \in \omega$ let $\mathcal{A}_{n}$ be a maximal antichain below $p\lceil\beta$ such that

(1) $\forall r \in \mathcal{A}_{n} \bar{r}$ is compact,

(2) $\forall r \in \mathcal{A}_{n} \exists t \subseteq \prod_{j<n} f(j) r \Vdash_{\mathbb{E E}_{\beta}} p(\beta)\lceil n=t$.

Fix a sequence $\left\langle F_{n}: n \in \omega\right\rangle$ such that for $n \in \omega$,

(1) $F_{n} \in[\beta]^{<\omega}$,

(2) $F_{n} \subseteq F_{n+1}$,

(3) $\bigcup_{n} F_{n}=\beta$.

By induction build a sequence $\left\langle q_{n}: n \in \omega\right\rangle$ such that for $n \in \omega$,

(1) $\overline{q_{n}}$ is compact,

(2) $q_{n+1} \geq_{F_{n}, n} q_{n}$,

(3) $\exists \mathcal{A}_{n}^{\prime} \in\left[\mathcal{A}_{n}\right]^{<\omega} \overline{q_{n}} \subseteq \bigcup_{r \in \mathcal{A}_{n}^{\prime}} \bar{r}$.

Let $q_{\omega}=\lim _{n} q_{n}$. As in the proof of Lemma[7we show that there exists a continuous function $F: \overline{q_{\omega}} \longrightarrow \mathbb{E} \mathbb{E}$ (encode elements of $\mathbb{E} \mathbb{E}$ as reals) such that

$$
q_{\omega} \Vdash_{\mathbb{E E}_{\beta}} p(\beta)=F\left(\left\langle\dot{g}_{\gamma}: \gamma<\beta\right\rangle\right) .
$$

Consider $q=q_{\omega} \frown p(\beta) \geq p$. Clearly, $\bar{q}=\left\{\langle x, y\rangle: x \in \overline{q_{\omega}}, y \in[F(x)]\right\}$ is compact in $\mathbf{X}^{\alpha}$. Remaining requirements are met as well. 
CASE 2. $\alpha$ is limit.

Given $p \in \mathbb{E E}_{\alpha}$ fix sequences $\left\langle F_{n}: n \in \omega\right\rangle$ and $\left\langle\alpha_{n}: n \in \omega\right\rangle$ such that

(1) $F_{n} \in\left[\alpha_{n}\right]^{<\omega}$,

(2) $F_{n} \subseteq F_{n+1}$,

(3) $\bigcup_{n} F_{n}=\alpha$,

(4) $\sup _{n} \alpha_{n}=\alpha$.

By induction build a sequence $\left\langle q_{n}: n \in \omega\right\rangle$ such that for $n \in \omega$,

(1) $q_{n} \in \mathbb{E E}_{\alpha}$,

(2) $\operatorname{supp}\left(q_{n}\right) \subseteq \alpha_{n}$

(3) $q_{n+1} \geq_{F_{n}, n} q_{n}$,

(4) $q_{n}\left\lceil\alpha_{n} \geq p\left\lceil\alpha_{n}\right.\right.$

(5) $\frac{q_{n}\left\lceil\alpha_{n}\right.}{q_{n}}$ is compact in $\mathbf{X}^{\alpha_{n}}$.

Let $q=\lim _{n} q_{n}$. Note that $\bar{q}=\bigcap_{n} \overline{q_{n}\left\lceil\alpha_{n}\right.} \times \mathbf{X}^{\alpha \backslash \alpha_{n}}$ is as required.

From now on we will always work with conditions $p$ such that $\bar{p}$ is good. We noticed earlier that for every condition $p \in \mathbb{E} \mathbb{E},[p]$ is canonically isomorphic to $2^{\omega}$. In exactly the same way we can verify that if $p \in \mathbb{E E}_{\alpha}$ and $\bar{p}$ is good, then $\bar{p}$ is isomorphic to $\left(2^{\omega}\right)^{\alpha}$.

As in Lemma 7 we show that:

Lemma 11. Suppose that $p \in \mathbb{E E}_{\alpha}$ and $p \Vdash_{\mathbb{E}_{\alpha}} \dot{x} \in 2^{\omega}$. Then there exist $q \geq p$ and a continuous function $F: \bar{p} \longrightarrow 2^{\omega}$ such that $q \Vdash_{\mathbb{E E}_{\alpha}} \dot{x}=F(\dot{\mathrm{g}})$, where $\dot{\mathbf{g}}=$ $\left\langle\dot{g}_{\beta}: \beta<\alpha\right\rangle$ is the sequence of generic reals.

Lemma 12. Let $p \in \mathbb{E}_{\alpha}$ and suppose that $H \subseteq \bar{p}$ is a meager set in $\bar{p}$. For every $F \in[\alpha]^{<\omega}$ and $n \in \omega$ there exists $q \geq_{F, n} p$ such that $\bar{q} \cap H=\emptyset$.

Proof. As before, without loss of generality we can assume that $\alpha$ is countable.

Induction on $\alpha$.

CASE 1. $\alpha=\beta+1$.

Suppose that $p \in \mathbb{E E}_{\alpha}$ and $H \subseteq \bar{p} \subseteq \mathbf{X}^{\beta} \times \mathbf{X}$ is meager, and let $F \in[\alpha]^{<\omega}$ and $n \in \omega$ be given.

Let

$$
H^{\prime}=\left\{x \in \overline{p\lceil\beta}:(H)_{x} \text { is not meager in }[p(\beta)[x]]=\left((\bar{p})_{x}\right)_{\beta}\right\} .
$$

Using the fact that $\bar{p}$ is homeomorphic to $\left(2^{\omega}\right)^{\alpha}$ via homeomorphism respecting vertical sections, and by Kuratowski-Ulam theorem, we conclude that $H^{\prime}$ is a meager set in $\overline{p\lceil\beta}$.

Recall the following classical lemma:

Lemma 13 ([1]). Suppose that $H \subseteq 2^{\omega} \times 2^{\omega}$ is a Borel set.

(1) Assume $(H)_{x}$ is meager for all $x$. Then there exists a sequence of Borel sets $\left\{G_{n}: n \in \omega\right\} \subseteq 2^{\omega} \times 2^{\omega}$ such that

(a) $\left(G_{n}\right)_{x}$ is a closed nowhere dense set for all $x \in 2^{\omega}$,

(b) $H \subseteq \bigcup_{n \in \omega} G_{n}$.

By the inductive hypothesis we can find $q^{\star} \geq_{F \cap \beta, n} p\left\lceil\beta\right.$ such that $\overline{q^{\star}} \cap H^{\prime}=\emptyset$. By Lemma [ for every $x \in \overline{q^{\star}}$ there exists $q_{x} \geq_{n} p(\beta)[x]$ such that $\left[q_{x}\right] \cap(H)_{x}=\emptyset$. Moreover, by Lemma 13, the mapping $x \mapsto q_{x}$ can be chosen to be Borel, and subsequently, by shrinking $q^{\star}$, continuous. Let $q \in \mathbb{E E}_{\alpha}$ be defined such that $q\left\lceil\beta=q^{\star}\right.$ and $q^{\star} \vdash_{\mathbb{E E}_{\beta}} q(\beta)=q_{\dot{g}_{\beta}}$. It is clear that $q$ has the required properties. 
CASE 2. $\alpha$ is limit.

Fix sequences $\left\langle F_{n}: n \in \omega\right\rangle$ and $\left\langle\alpha_{n}: n \in \omega\right\rangle$ such that

(1) $F_{n} \in\left[\alpha_{n}\right]^{<\omega}$,

(2) $F_{n} \subseteq F_{n+1}$,

(3) $\bigcup_{n} F_{n}=\alpha$,

(4) $\sup _{n} \alpha_{n}=\alpha$.

By induction build a sequence $\left\langle q_{n}: n \in \omega\right\rangle$ such that for $n \in \omega$,

(1) $q_{n} \in \mathbb{E E}_{\alpha}$,

(2) $\operatorname{supp}\left(q_{n}\right) \subseteq \alpha_{n}$

(3) $q_{n+1} \geq_{F_{n}, n} q_{n}$,

(4) $q_{n}\left\lceil\alpha_{n} \geq p\left\lceil\alpha_{n}\right.\right.$,

(5) $\overline{q_{n}\left\lceil\alpha_{n}\right.} \cap H_{n}=\emptyset$, where $H_{n}=\left\{x \in \overline{q_{n}\left\lceil\alpha_{n}\right.}:(H)_{x}\right.$ is not meager in $\left.\overline{p[x]}\right\}$.

As before (5) is possible by Kuratowski-Ulam theorem. Let $q=\lim _{n} q_{n}$. It is clear that $\bar{q} \cap H=\emptyset$.

The following lemma is an analog of Lemma 8

Lemma 14. Suppose that $p \in \mathbb{E}_{\alpha}, n \in \omega$ and $p \Vdash_{\mathbb{E E}_{\alpha}} \dot{x} \in 2^{\omega}$. Let $F: \bar{p} \longrightarrow 2^{\omega}$ be a continuous function such that $p \Vdash_{\mathbb{E E}_{\alpha}} \dot{x}=F(\dot{\mathbf{g}})$, where $\dot{\mathbf{g}}=\left\langle\dot{g}_{\beta}: \beta<\alpha\right\rangle$ is the sequence of generic reals. There exists $q \geq p$ such that exactly one of the following conditions holds:

(1) $F\lceil\bar{q}$ is constant,

(2) there exists $\beta<\alpha$ such that $F\lceil\overline{q \uparrow \beta}$ is one-to-one and for every $x \in \overline{q \uparrow \beta}$, $F \uparrow(\overline{q \uparrow \beta})_{x}$ is constant,

(3) $F\lceil\bar{q}$ is one-to-one.

Proof. We have three cases:

CASE 1. There exists $q \geq p$ such that $q \Vdash_{\mathbb{E E}_{\alpha}} \dot{x} \in \mathbf{V}$. Without loss of generality we can assume that for some $x \in \mathbf{V} \cap 2^{\omega}, q \Vdash_{\mathbb{E E}_{\alpha}} \dot{x}=x$. It follows that $F\lceil\bar{q}$ is constant.

CASE 2. There exists $q \geq p$ such that $q \Vdash_{\mathbb{E E}_{\alpha}} \exists \beta<\alpha \dot{x} \in \mathbf{V}^{\mathbb{E E}_{\beta}}$. By shrinking $q$ we can assume that there exists a continuous function $G: \overline{q \uparrow \beta} \longrightarrow 2^{\omega}$ such that $q \Vdash_{\mathbb{E}_{\alpha}} \dot{x}=G(\dot{\mathbf{g}}\lceil\beta)$. In particular, for $x \in[q], F(x)=G(x\lceil\beta)$. If $\beta$ was minimal, then, using the argument below, we can also assume that $G$ is one-to-one.

Suppose that $q \in \mathbb{E E}_{\alpha}, F \in[\alpha]^{<\omega}$, and $n \in \omega$. Without loss of generality we can assume that for every $\beta \in F, q\left\lceil\beta\right.$ determines the value of $\operatorname{split}_{n}(q(\beta)$ ) (up to finitely many values). Suppose that $\sigma: F \longrightarrow \omega^{<\omega}$ is a function such that $\sigma(\beta) \in \operatorname{split}_{n}(q(\beta))$ for $\beta \in F$. Let $(q)_{\sigma}$ be the condition defined as

$$
\forall \beta<\alpha(q)_{\sigma}\left\lceil\beta \Vdash_{\mathbb{E E}_{\beta}}(q)_{\sigma}(\beta)= \begin{cases}q(\beta) & \text { if } \beta \notin F, \\ (q(\beta))_{\sigma(\beta)} & \text { if } \beta \in F .\end{cases}\right.
$$

Let $\Sigma_{F, n}$ be the finite set of all mappings $\sigma$ satisfying the requirements.

Lemma 15. Suppose that $F \in[\alpha]^{<\omega}, n \in \omega$ and

$$
p \Vdash_{\mathbb{E E}_{\alpha}} \dot{x}=F(\dot{\mathbf{g}}) \& \forall \beta<\alpha \dot{x} \notin \mathbf{V}^{\mathbb{E E}_{\beta}} .
$$

There exists $q \geq_{F, n} p$ such that the sets $\left\{F^{\prime \prime}\left(\overline{(q)_{\sigma}}\right): \sigma \in \Sigma_{F, n}\right\}$ are pairwise disjoint. 
Proof. Induction on $|F|$ and $\alpha$. If $F=\{\beta\}$ this is essentially Lemma 8

Let $\left\{v_{j}: j<k^{\star}\right\}$ be an enumeration of $\operatorname{split}_{n}(p(\beta))$. For $v \in \operatorname{split}_{n}(p)$ choose pairwise different reals $x_{v} \in F^{\prime \prime}\left(\overline{(p)_{v}}\right)$. Note that this choice can be made canonically from, for example, the countable dense set of leftmost branches of subtrees of $p$. Let $\ell>k$ be such that sequences $x_{v} \mid \ell$ are also pairwise different. Define conditions $\left\langle r_{j}: j \leq k^{\star}\right\rangle,\left\langle q_{j}: j \leq k^{\star}\right\rangle$ such that for every $j \leq k^{\star}$,

(1) $r_{j} \in \mathbb{E}_{\beta}$,

(2) $r_{j+1} \geq r_{j}$

(3) $r_{j} \Vdash_{\mathbb{E E}_{\beta}} q_{j} \geq(p)_{v_{j}} \uparrow[\beta, \alpha)$,

(4) $\forall z \in \overline{r_{j} \frown q_{j}}, F(z)\left\lceil\ell=F\left(x_{v_{j}}\right)\lceil\ell\right.$.

Let $q \uparrow \beta=q_{k^{\star}}$ and $q \uparrow[\beta, \alpha)=\bigcup_{j<k^{\star}} q_{j}$.

Suppose that $|F|=k+1$ and let $\beta=\max (F)$.

By the part already proved, for each $\mathbf{x}=\left\langle x_{\gamma}: \gamma\langle\beta\rangle \in \overline{p\lceil\beta}\right.$ find a condition $q_{\mathbf{x}} \geq_{n} p\left\lceil[\beta, \alpha)[\mathbf{x}]\right.$ such that the sets $\left\{F^{\prime \prime}\left(\overline{\left(q_{\mathbf{x}}\right)_{s}}\right): s \in\right.$ split $\left._{n}\left(q_{\mathbf{x}}\right)\right\}$ are pairwise disjoint. Note that we can do it in such a way that the mapping $\mathbf{x} \mapsto q_{\mathbf{x}}$ is continuous. (As before we first choose $q_{\mathbf{x}}$ in a Borel way, and then shrink $p\lceil\beta$ to make this mapping continuous.) That defines an $\mathbb{E}_{\beta}$-name for an element of $\mathbb{E}_{\beta, \alpha}$, which we call $q^{\star}$.

Next, let $F^{\prime}=F \backslash\{\beta\}$ and apply the inductive hypothesis to find $q^{\prime} \geq_{F^{\prime}, n} p\lceil\beta$ such that $\left\{F^{\prime \prime}\left(\overline{\left(q^{\prime}\right)_{\sigma}}\right): \sigma \in \Sigma_{F^{\prime}, n}\right\}$ are pairwise disjoint. Let $q \in \mathbb{E}_{\alpha}$ be defined as $q \uparrow \beta=q^{\prime}$ and $q\left\lceil\beta \Vdash_{\mathbb{E E}_{\beta}} q \uparrow[\beta, \alpha)=q^{\star}\right.$.

It is clear that $q$ is as required.

CASE 3. $p \Vdash_{\mathbb{E E}_{\alpha}} \forall \beta<\alpha \dot{x} \notin \mathbf{V}^{\mathbb{E E}_{\beta}}$.

Let $\left\langle F_{n}: k \in \omega\right\rangle$ be an increasing sequence of finite sets such that $\bigcup_{n} F_{n}=\alpha$.

By induction build a sequence of conditions $\left\langle p_{n}: n \in \omega\right\rangle$ such that $p_{0}=p$ and for every $n$,

(1) $p_{n+1} \geq_{F_{n}, n} p_{n}$

(2) $\operatorname{sets}\left\{F^{\prime \prime}\left(\overline{\left.\left(p_{n}\right)_{\sigma}\right)}: \sigma \in \Sigma_{F_{n}, n}\right\}\right.$ are pairwise disjoint.

Let $q=\lim _{n} p_{n}$.

Suppose that $\mathbf{x}=\left\langle x_{\beta}: \beta<\alpha\right\rangle$ and $\mathbf{x}^{\prime}=\left\langle x_{\beta}^{\prime}: \beta<\alpha\right\rangle$ are two distinct points in $\bar{q}$. Let $\beta$ be the first ordinal such that $x_{\beta} \neq x_{\beta}^{\prime}$. Let $n$ be so large that $\beta \in F_{n}$ and there are two distinct $\sigma, \sigma^{\prime} \in \Sigma_{F_{n}, n}$ such that $\mathbf{x} \in \overline{\left(p_{n}\right)_{\sigma}}$ and $\mathbf{x}^{\prime} \in \overline{\left(p_{n}\right)_{\sigma^{\prime}}}$. Since $F^{\prime \prime}\left(\overline{\left(p_{n}\right)_{\sigma}}\right) \cap F^{\prime \prime}\left(\overline{\left(p_{n}\right)_{\sigma^{\prime}}}\right)=\emptyset$, it follows that $F(\mathbf{x}) \neq F\left(\mathbf{x}^{\prime}\right)$.

\section{A MOdel WHERE $\mathbf{P M} \subseteq \mathbf{U N}$}

Let $\mathbb{E E}_{\omega_{2}}$ be the countable support iteration of $\mathbb{E} \mathbb{E}$ of length $\aleph_{2}$. We will show that in $\mathbf{V}^{\mathbb{E E}_{\omega_{2}}}, \mathbf{P M} \subseteq \mathbf{U N}$.

By Theorem $9(2), \mathbf{V}^{\mathbb{E}_{\omega_{2}}} \models[\mathbb{R}]^{<2^{\aleph_{0}}} \subseteq \mathbf{U N}$, thus we have to show that

$$
\mathbf{V}^{\mathbb{E E}_{\omega_{2}}}=\mathbf{P M} \subseteq[\mathbb{R}]^{<2^{\aleph_{0}}} .
$$

Suppose that $X \in \mathbf{V}^{\mathbb{E E}_{\omega_{2}}}$ is a set of reals of size $\boldsymbol{\aleph}_{2}$. Let $\left\{\dot{x}_{\alpha}: \alpha<\omega_{2}\right\}$ be the set of names for elements of $X$ such that $\mathbb{E}_{\mathbb{E}_{\omega_{2}}} \forall \alpha \neq \beta \dot{x}_{\alpha} \neq \dot{x}_{\beta}$. Apply Lemma 11 and find for each $\alpha<\omega_{2}$ a set $w_{\alpha} \in\left[\omega_{2}\right]^{\leq \omega}$, a condition $p_{\alpha} \in \mathbb{E E}_{w_{\alpha}}$, and a continuous 
function $F_{\alpha}: \overline{p_{\alpha}} \longrightarrow 2^{\omega}$ such that $p_{\alpha} \Vdash_{\mathbb{E}_{\mathbb{E}_{2}}} \dot{x}_{\alpha}=F_{\alpha}\left(\left\langle\dot{g}_{\beta}: \beta \in w_{\alpha}\right\rangle\right)$. We can assume that $w_{\alpha}$ is minimal. In other words, $p_{\alpha} \Vdash_{\mathbb{E E}_{\omega_{2}}} \forall \beta<\sup \left(w_{\alpha}\right) \dot{x}_{\alpha} \notin \mathbf{V}^{\mathbb{E} \mathbb{E}_{\beta}}$. In particular, without loss of generality we can assume $F_{\alpha}$ is one-to-one, so it is a homeomorphism.

By thinning out we can assume that ot $\left(w_{\alpha}\right)=\gamma, F_{\alpha}=F$ and $\overline{p_{\alpha}}=\bar{p}$. Moreover, since $\mathbf{V}=\mathrm{CH}$, we can assume that $w_{\alpha} \cap w_{\beta}=w^{\star}$ for $\alpha \neq \beta$. Finally, without loss of generality we can assume that $w^{\star}=\emptyset$.

Let $P=F^{\prime \prime}(\bar{p})$. Since $F$ is a homeomorphism, $P$ is perfect. We will show that $X \cap P$ is not meager in $\mathbf{V}^{\mathbb{E E}_{\omega_{2}}}$ (relative to $P$ ).

Assume otherwise and let $H \subseteq P$ be a meager set such that for some $p^{\star} \in \mathbb{E E}_{\omega_{2}}$, $p^{\star} \Vdash_{\mathbb{E E}_{\omega_{2}}} X \cap P \subseteq H$. By Theorem 9(4) we can assume that $H \in \mathbf{V}$. Set $G=$ $(F)^{-1}(H)$ and notice that $G$ is a meager subset of $\bar{p}$.

Find $\alpha<\omega_{2}$ such that $w_{\alpha} \cap \mathrm{cl}\left(p^{\star}\right)=\emptyset$. By Lemma 12 there exists $q \geq p$, $q \in \mathbb{E E}_{w_{\alpha}} \simeq \mathbb{E E}_{\gamma}$ such that $\bar{q} \cap G=\emptyset$.

Since $p^{\star}$ and $q$ are compatible let $r \geq p^{\star}, q$. It follows that

$$
r \Vdash_{\mathbb{E E}_{\omega_{2}}} \dot{x}_{\alpha}=F_{\alpha}\left(\left\langle\dot{q}_{\beta}: \beta \in w_{\alpha}\right\rangle\right) \notin H,
$$

which finishes the proof.

\section{ACKNowledgments}

The work was done while the first author was spending his sabbatical year at Rutgers University and the College of Staten Island, CUNY. Their support is gratefully acknowledged.

\section{REFERENCES}

[1] Tomek Bartoszyński. Measure and Category Invariants. In M. Magidor, M. Foreman, A. Kanamori, editors, Handbook of Set Theory. submitted.

[2] Tomek Bartoszynski. On perfectly meager sets. Proceedings of the American Mathematical Society, 130(4):1189-1195, 2002. CMP 2002:06

[3] Tomek Bartoszyński and Haim Judah. Set Theory: on the structure of the real line. A.K. Peters, 1995. MR 96k:03002

[4] Paul Corazza. The generalized Borel conjecture and strongly proper orders. Transactions of the American Mathematical Society, 316(1):115-140, 1989. MR 90c:03042

[5] Martin Goldstern, Haim Judah, and Saharon Shelah. Strong measure zero sets without Cohen reals. The Journal of Symbolic Logic, 58(4):1323-1341, 1993. MR 95c:03126

[6] Edward Grzegorek. Solution of a problem of Banach on $\sigma$-fields without continuous measures. L'Academie Polonaise des Sciences. Bulletin. Serie des Sciences Mathematiques, 28(1-2):710, 1980. MR 82h:04005

[7] Arnold W. Miller. Some properties of measure and category. Transactions of the American Mathematical Society, 266(1):93-114, 1981. MR 84e:03058a

[8] Arnold W. Miller. Mapping a set of reals onto the reals. The Journal of Symbolic Logic, 48(3):575-584, 1983. MR 84k:03125

[9] Arnold W. Miller. Special subsets of the real line. In K. Kunen and J. E. Vaughan, editors, Handbook of Set Theoretic Topology, pages 201-235. North-Holland, Amsterdam, 1984. MR 86i:54037

[10] Andrzej Rosłanowski and Saharon Shelah. Norms on possibilities I: forcing with trees and creatures. Memoirs of the American Mathematical Society. American Mathematical Society, 1999. MR 2000c:03036

[11] Saharon Shelah. Proper and Improper Forcing. Perspectives in Logic. Springer-Verlag, 1998. MR 98m:03002 
[12] Waclaw Sierpiński. Sur une probleme de M. Kuratowski concernant la propriete de Baire des ensambles. Fundamenta Mathematicae, 22:262-266, 1934.

[13] Piotr Zakrzewski. Universally meager sets. Proceedings of the American Mathematical Society, 129(6):1793-1798, 2001. MR 2001m:03097

Department of Mathematics and Computer Science, Boise State University, Boise, IDAHO 83725

E-mail address: tomek@math.boisestate.edu

$U R L:$ http://math.boisestate.edu/ ${ }^{\text {tomek }}$

Department of Mathematics, Hebrew University, Jerusalem, Israel - and - Department of Mathematics, Rutgers University, New Brunswick, New Jersey

E-mail address: shelah@math.huji.ac.il

URL: http://math.rutgers.edu/ ${ }^{\sim}$ shelah/ 